\title{
USO DA PROGRAMAÇÃO LINEAR INTEIRA PARA A FORMULAÇÃO DE UMA APÓLICE DE SEGURO DE VIDA: UMA ABORDAGEM CIENTÍFICA
}

\author{
Igor Feitosa Pereira \\ Centro Universitário Augusto Motta - UNISUAM \\ Rua Fonseca, 240 - Bangu - Rio de Janeiro - RJ \\ igorfeitosa16@gmail.com \\ Ernesto Rademaker Martins \\ Centro de Análises de Sistemas Navais - CASNAV \\ Ed. 23 do AMRJ - R. da Ponte, s/n - Centro, Rio de Janeiro - RJ, 20091-000 \\ rademaker@casnav.mar.mil.br
}

\begin{abstract}
RESUMO
Este artigo tem como objetivo montar uma apólice de seguro de vida, com o menor custo possível, de forma que supra as necessidades de um cliente de uma corretora localizada na Barra da Tijuca, Zona Oeste do Rio de Janeiro. Para isso, utilizará uma técnica da Pesquisa Operacional, a Programação Linear Inteira, respaldando cientificamente a sua conjuntura. Como, no Brasil, o mercado de seguros de vida é bastante limitado, as empresas devem sempre inovar os seus métodos para manter, ou até aumentar, a sua participação, e este trabalho propõe a PLI como uma alternativa de inovação para a jovem organização. Com atuação abrangente, a Pesquisa Operacional, utilizada em várias áreas, como o setor industrial, comércio, esportes, entre outras, demonstra, outra vez, a sua efetividade.
\end{abstract}

Palavras-chave: Pesquisa Operacional, Programação Linear Inteira, Seguros de Vida.

\begin{abstract}
The paper has the objective to build a life insurance policy, with the lowest possible cost, supplying the necessities of a client of a broker based on Barra da Tijuca, West Side of the city of Rio de Janeiro. For this purpose, a Operational Research technique, Integer Linear Programming, will be used, scientifically supporting its conjecture. Since the life insurance Market is quite limited, companies must often innovate their methods, and this work proposes Integer Linear Programming as an alternative for innovation in this recently open organization. With its comprehensive performance, Operational Research, which is commonly used in several areas, such as the industrial sector, commerce, sports, among ohers, demonstrates, once again, its effectiveness.
\end{abstract}

Keywords: Operational Research, Integer Linear Programming, Life Insurance.

\section{Como Citar:}

PEREIRA, Igor Feitosa; MARTINS, Ernesto Rademaker. Uso da programação linear inteira para a formulação de uma apólice de seguro de vida: uma abordagem científica.. In: SIMPÓSIO DE PESQUISA OPERACIONAL E LOGÍSTICA DA MARINHA, 19., 2019, Rio de Janeiro, RJ. Anais [...]. Rio de Janeiro: Centro de Análises de Sistemas Navais, 2019. 


\section{INTRODUÇÃO}

Em um mundo corporativo cada vez mais acirrado, em que há uma ampla diversidade de ofertas, as empresas necessitam se reinventar constantemente, independentemente da área de atuação. Para isso, essas organizações devem buscar novos métodos e ferramentas para a melhoria dos seus produtos ou serviços. Quando se trata de um mercado com uma demanda, relativamente, menor, como os seguros de vida, essa necessidade é ainda mais latente.

A Pesquisa Operacional (PO) é capaz de suprir essa necessidade, por ser, segundo Santos et al (2016) uma ferramenta de otimização por excelência. Tendo surgido em 1939, durante a II Guerra Mundial, a PO tem uma gama de aplicações que vão desde manufatura, transportes, construção, telecomunicações, planejamento financeiro, assistência médica, militar até serviços públicos, dentre outras (HILLIER; LIEBERMAN, 2010).

Umas das técnicas mais conhecidas da Pesquisa Operacional é a Programação Linear (PL), que, de acordo com Santos et al (2017) por se tratar de um modelo simples, baseado em equações lineares, pode ser programável em computador, o que a torna uma ferramenta fácil de ser utilizada em qualquer tomada de decisão.

O objetivo do presente trabalho é montar uma apólice de seguro de vida para um cliente com o menor custo possível, de forma que corresponda à necessidade do mesmo, por meio da Programação Linear Inteira. Como ferramenta de processamento computacional foi utilizado o software Linear Interactive Discrete Optimizer (LINDO).

\section{FUNDAMENTAÇÃO TEÓRICA}

\subsection{SEGUROS DE VIDA}

O mercado de Seguros de Vida no Brasil ainda engatinha. Segundo Sutto (2017) um estudo feito pela Universidade de Oxford, em conjunto com a seguradora Zurich, revelou que apenas $19 \%$ da população brasileira possui algum tipo de cobertura em caso de morte, assim sendo, o pior índice entre os países estudados, $2 \%$ atrás do segundo pior, o Reino Unido. Em relação à doenças e invalidez, o número cresce um pouco, $22 \%$.

A Figura 1 mostra os dados obtidos pelo estudo:

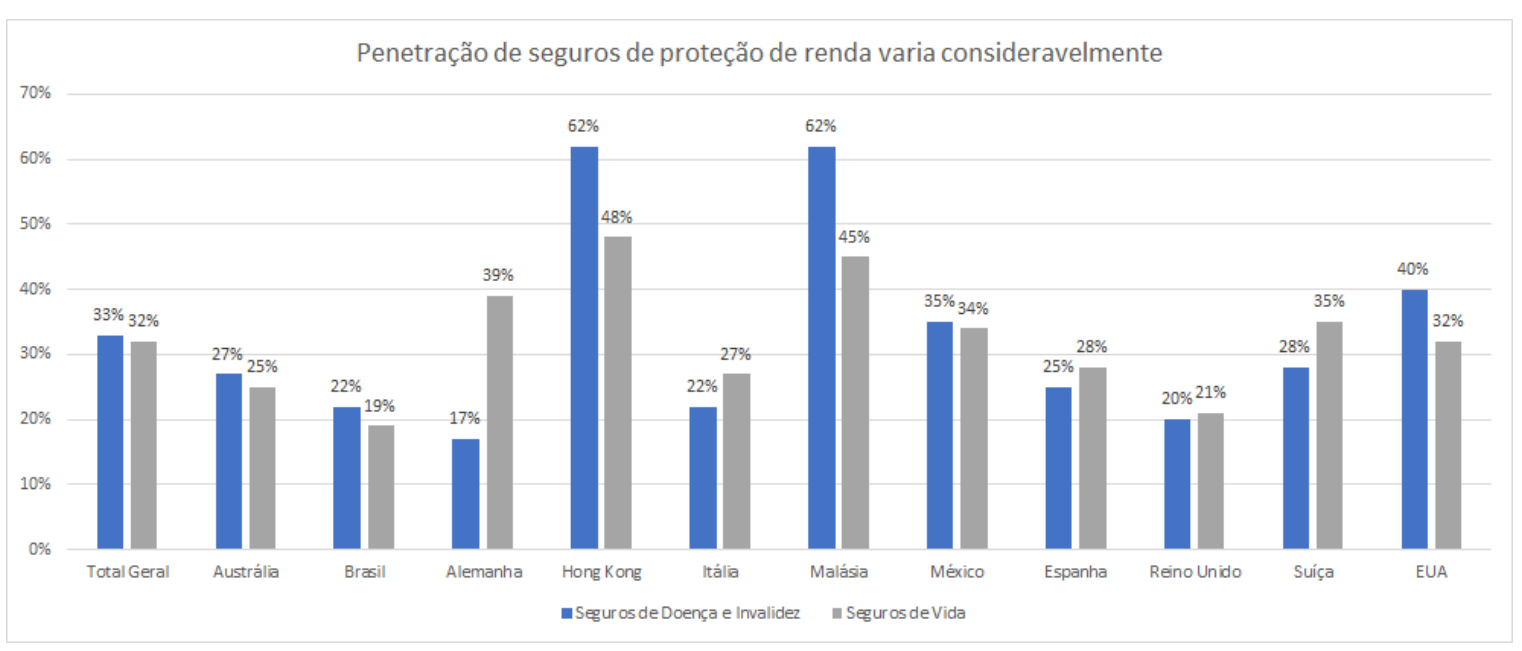

Figura 1 - Estudo internacional sobre seguros feito pela Universidade de Oxford Fonte: Sutto - Adaptado (2017) 


\subsection{PESQUISA OPERACIONAL} possuir

O uso da Pesquisa Operacional na problemática apresentada neste trabalho por diversas técnicas e modelos que estão inerentes à redução de custos, de forma a atender às expectativas do cliente, dando respaldo matemático para a análise.

Para Loesch e Hein (2009), a utilização da PO é uma maneira de trazer conhecimento para uma organização, uma vez que ela age como ferramenta de análise e estudo das problemáticas dos processos organizacionais.

Segundo Milhomem et al (2015) a pesquisa Operacional (PO) surgiu, na Segunda Guerra Mundial, como resultado de estudos de equipes interdisciplinares de cientistas contratados para resolver problemas militares de ordem estratégica e tática.

Apesar de sua razão de ser, a PO tem sido mais utilizada no meio coorporativo, com enfoque no industrial e produção, do que para fins militares, o que, para Hillier e Lieberman (2013) se dá a não incidência de guerras como no período em que foi criada.

Santos (2013) destaca que a PO lança mão de modelos matemáticos e/ou lógicos, a fim de resolver problemas reais, apresentando um caráter eminentemente multidisciplinar. Assim sendo, de acordo com o tipo e com a complexidade do problema a ser estudado, serão escolhidos os melhores modelos que aderem àquela realidade.

\subsection{PROGRAMAÇÃO LINEAR}

A PL é um tópico da ciência da PO e é definida como uma técnica de planejamento baseada em matemática e economia que, aliada à computação, teve seu desenvolvimento e difusão acelerados. A PL é uma técnica de otimização. Além disso, a PL é uma ferramenta utilizada para encontrar o lucro máximo ou o custo mínimo em situações nas quais tem-se diversas alternativas de escolha, sujeitas a algum tipo de restrição ou regulamentação (REZENDE FILHO, 2006).

O seu modelo básico é composto por uma Função Objetivo (FO), restrições técnicas e de não negatividade, como mostra a Figura 2. Para Silva et al (2007), "a FO afere a execução do modelo e as restrições, por sua vez, garantem que as limitações impostas no sistema sejam respeitadas." 


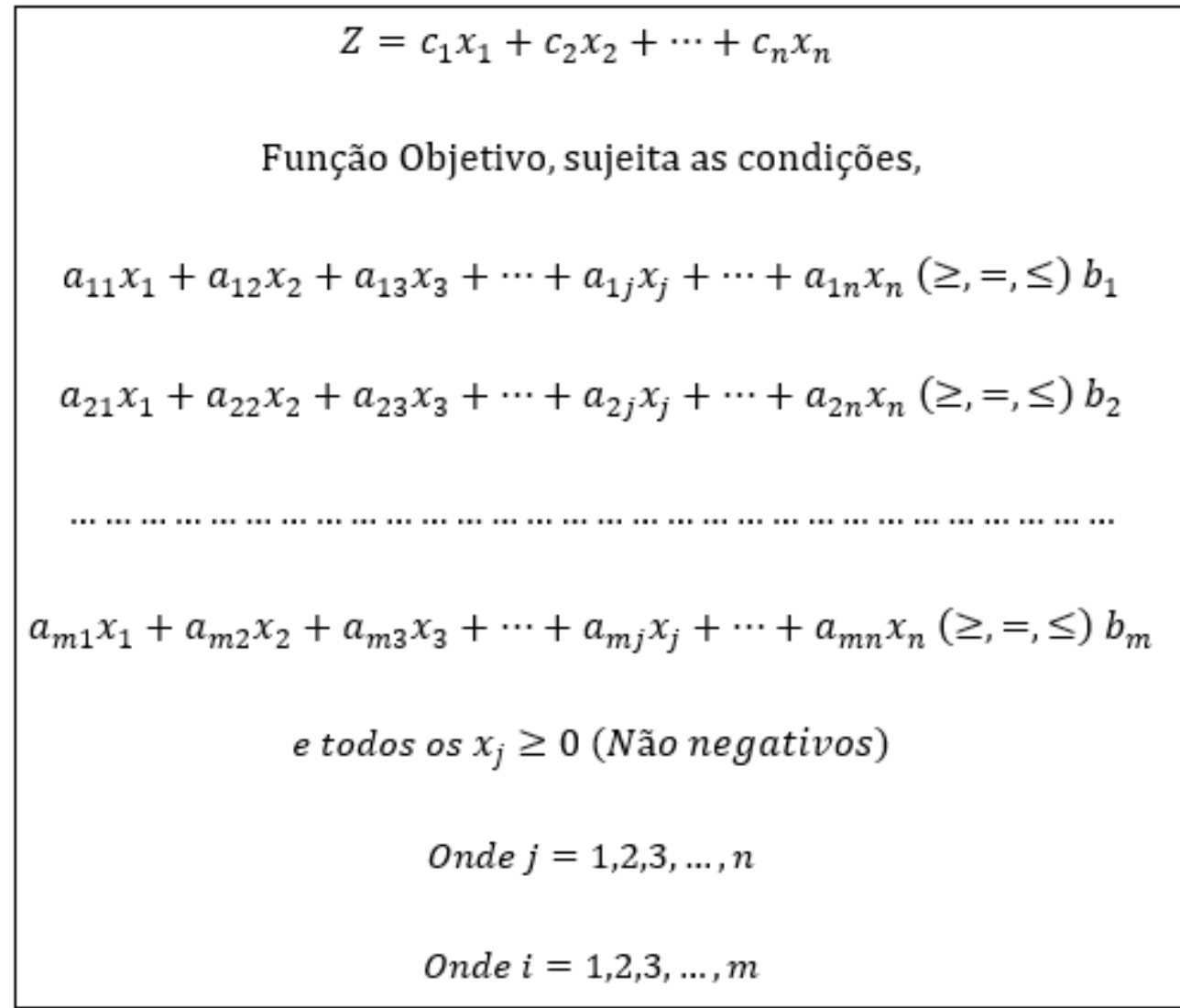

Figura 2 - Modealgem básica da PL Fonte: MURTHY (2005)

Barbosa (2014) diz que "problema de otimizar uma função linear sujeita a restrições teve a sua origem com os estudos de Fourier sobre sistemas lineares de inequações em 1826. No entanto só em 1939 Kantorovich faz notar a importância prática destes problemas, tendo criado um algoritmo para a sua solução. O auge do estudo de problemas de otimização de uma função linear sujeita a restrições foi com George Dantzig na década de 1940, nos Estados Unidos. Dantzig não só formulou o problema de programação linear, mas também criou o algoritmo do Simplex em 1947”.

\subsection{PROGRAMAÇÃO LINEAR INTEIRA}

A PLI possui uma estrutura bastante similar à PL, porém, segundo Colin (2011) ela inclui uma restrição ao problema: de que pelo menos uma das variáveis da solução ótima seja pertencente ao conjunto dos números inteiros. Já Taha (2008) afirma que todas as variáveis precisam ser inteiras, caso contrário, constitui-se em uma Programação Linear Inteira Mista.

$\mathrm{O}$ algoritmo mais usado para resolução da PLI é o Branch and Bound (B\&B). Segundo o Instituto de Ciências Matemáticas e Computação da Universidade de São Paulo (2011) "O método Branch-and-Bound (B\&B) baseia-se na idéia de desenvolver uma enumeração inteligente das soluções candidatas à solução ótima inteira de um problema.".

Santos et al (2017) descreveram o método Branch and Bound de forma gráfica, conforme mostra a Figura 3. 


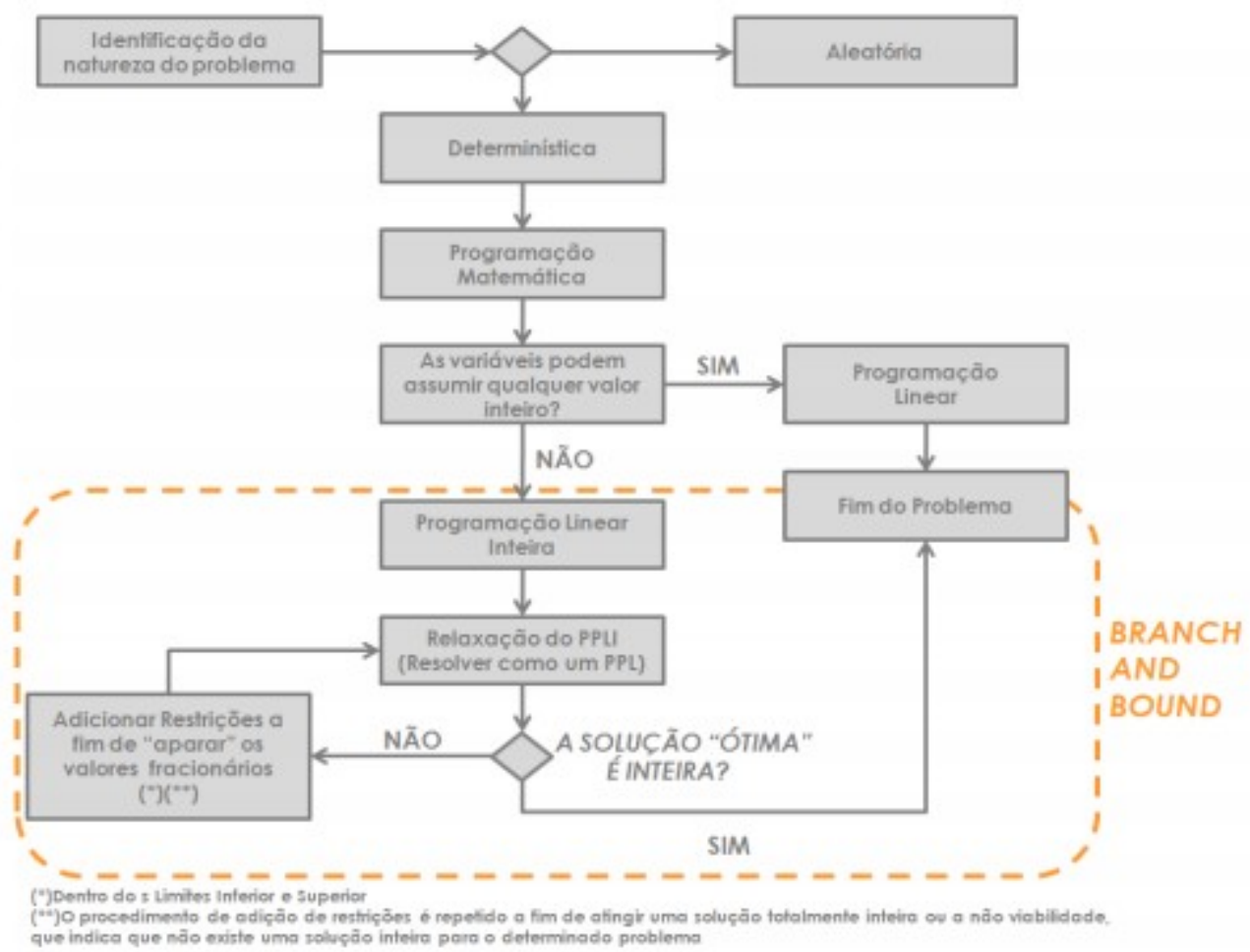

Figura 3 - Esquema do Método Branch and Bound

Fonte: Santos et al (2017)

Foi realizada uma pesquisa do termo "Integer Linear Programming” (Programação Linear Inteira, em inglês) no portal de periódicos da Comissão de Aperfeiçoamento de Pessoal do Nível Superior (CAPES), afim de determinar o número do publicações sobre o tema entre os anos de 1998 e 2018, conforme mostra a Figura 4. Nota-se um claro aumento no número nos últimos 10 anos, o que demonstra um significativo aumento da relevância do assunto.

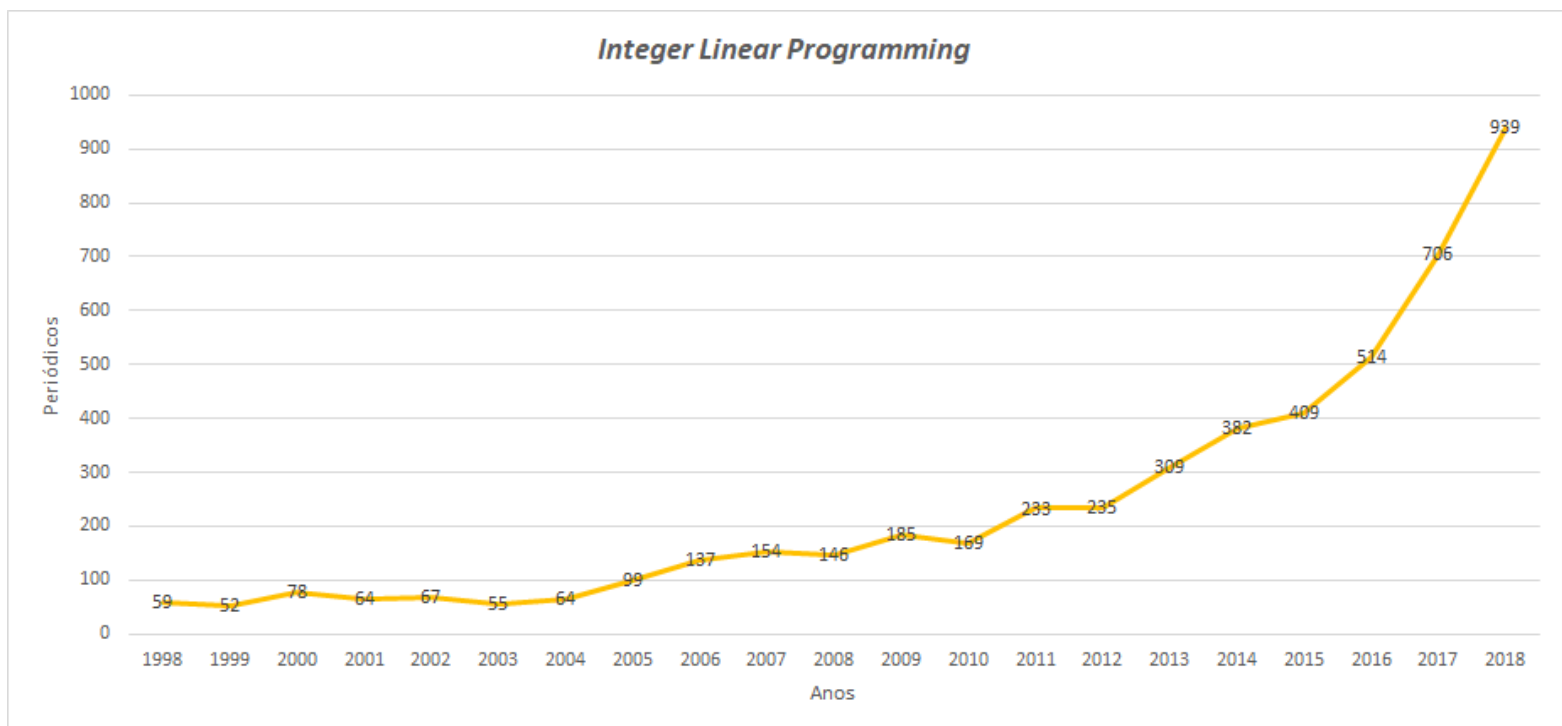

Figura 4 - Pesquisa realizada no portal da CAPES com o termo Integer Linear Programming Fonte: Autor (2019)

\section{METODOLOGIA}


Determinou-se como método de pesquisa o Estudo de Caso, método que se baseia na análise de episódio ou contexto específico, buscando a resolução de um problema que afeta a organização, seja coorporativa, com ou sem fins lucrativos, ou até mesmo social.

Para Santos et al (2017) todo problema de PO possui uma lógica simples e encadeada, onde se parte de uma situação problemática, isto é, uma incompatibilidade entre o que se quer e o que se tem.

Hillier e Lieberman (2010) corroboram ao apontar que existem seis fases na resolução através da Pesquisa Operacional. São eles:

1- Definir o problema a ser solucionado e coletar dados;

2- Criar um modelo matemático que represente o problema;

3- Desenvolver um procedimento computacional para que se encontre possíveis soluções a partir do modelo;

4- Testar o modelo e aprimorá-lo, caso precise;

5- Preparar-se para a aplicação contínua do modelo;

6- Implementá-lo.

A escolha da utilização da PLI justifica-se pela necessidade de uma quantidade de um determinado produto presente no modelo. Para isso, foi preciso entender o processo de vendas de seguros de vida e as regras e restrições impostas no produtos pela Seguradora MA e pelo cliente. Para a resolução do modelo foi utilizado o software LINDO v.6.1 trial version, da LINDO Systems, que é bem conhecido e confiável.

A empresa em questão foi fundada em 2018 e situa-se, no momento, na Zona Oeste da cidade do Rio de Janeiro.

\section{ESTUDO DE CASO}

O estudo de caso foi realizado em uma corretora de seguros carioca, situada no bairro da Barra da Tijuca. Apesar de nova e ser relativamente pequena, a empresa conta com profissionais com experiência no ramo e que já possuem uma carteira de clientes bem robusta.

O Cliente X procurou a corretora interessado em produtos de proteção em caso de morte, invalidez parcial ou total e doenças graves. Em uma primeira consulta, detectou-se o perfil: Homem, advogado, 40 anos, casado com um filho de 12 anos de idade e renda mensal de $\mathrm{R} \$ 23.000,00$ (vinte e três mil reais). O cliente não é fumante, se encontrava saudável e não fazia uso de qualquer tipo de medicamento regularmente ou praticava esportes radicais. Suas principais prioridades com o seguro são a educação do filho, que estuda em uma escola com mensalidade em torno de R \$ 3.000,00 (três mil reais), e que, em sua ausência, a família mantivesse o padrão de vida por tempo suficiente para se restabelecer.

\section{MODELAGEM MATEMÁTICA}

O objetivo do modelo é minimizar o custo mensal do cliente com o seguro, de forma que as necessidades sejam supridas.

A Tabela 1 mostra os produtos que, dentre os oferecidos pela Seguradora MA, a corretora comercializa. 


\begin{tabular}{|c|c|}
\hline \multicolumn{2}{|c|}{ Lista de produtos Seguradora MA } \\
\hline Whole Life com pagamento vitalício & Term Life de 30 anos \\
\hline Whole Life com pagamento por 10 anos & Morte Acidental \\
\hline Whole Life com pagamento por 15 anos & Diária por Internação Hospitalar (DIH) 150 \\
\hline Whole Life com pagamento por 20 anos & Diária por Internação Hospitalar (DIH) 200 \\
\hline Whole Life com pagamento por 25 anos 250 \\
\hline Whole Life com pagamento por 30 anos & Doraves \\
\hline Whole Life com pagamento até 60 anos & Diária por Incapacidade Temporária com LER e DORT (DIT) \\
\hline Whole Life com pagamento até 65 anos & Invalidez Permanente Total ou Parcial por Acidente (IPA) \\
\hline Whole Life com pagamento até 70 anos & Invalidez Funcional Permanente por Doença (IFPD) \\
\hline Whole Life com pagamento até 75 anos & Seguro de Assistência Funeral Luxo Individual \\
\hline Term Life de 10 anos & Seguro de Assistência Funeral Luxo Familiar \\
\hline Term Life de 15 anos & Seguro de Assistência Funeral Superluxo Individual \\
\hline Term Life de 20 anos & Seguro de Assistência Funeral Superluxo Familiar \\
\hline Term Life de 25 anos & \\
\hline
\end{tabular}

Tabela 1 - Produtos oferecidos pela corretora

Fonte: Autor (2019)

O Cliente $\mathrm{X}$ demonstrou interesse nos seguintes produtos: Whole Life com pagamento até 60 anos, Term Life de 20 anos, Doenças Graves, Diária por Incapacidade Temporária com LER e DORT (DIT), Invalidez Permanente Total ou Parcial por Acidente (IPA), Invalidez Funcional Permanente por Doença (IFPD). Também expôs o desejo no produto Seguro de Assistência Funeral, porém não determinou qual modalidade.

\subsection{FUNÇÃO OBJETIVO}

Para elaboração da Função Objetivo (FO), determinou-se o valor de pagamento por milhar de benefício de cada produto. Afim de facilitar a visualização, a Tabela 2 mostra os produtos com as suas respectivas cotas.

\begin{tabular}{|c|c|c|c|c|}
\hline $\begin{array}{l}\text { Variável de } \\
\text { decisão }\end{array}$ & Produto & Benefício & Custo & $\begin{array}{c}\text { Custo por } \mathrm{R} \$ 1.000,00 \\
\text { de benefício }\end{array}$ \\
\hline $\mathbf{x 1}$ & Whole Life com pagamento até 60 anos & $\mathrm{R} \$ 5^{2} 00.000,00$ & $\mathrm{R} \$ 1.313,04$ & 2,626 \\
\hline $\mathbf{x 2}$ & Term Life de 20 anos & $\mathrm{R} \$ 500.000,00$ & $\mathrm{R} \$ \mathbf{1} \quad 160,20$ & 0,320 \\
\hline x3 & Doenças Graves & $\mathrm{R} \$ 500.000,00$ & $\mathrm{R} \$ 225,24$ & 0,450 \\
\hline$x 4$ & Diária por Incapacidade Temporária com LER e DORT (DIT) & $\mathrm{R} \$ \quad 5.000,00$ & $\mathrm{R} \$ \mathbf{1 0 9 , 0 2}$ & 21,804 \\
\hline$x 5$ & Invalidez Permanente Total ou Parcial por Acidente (IPA) & $\mathrm{R} \$ 500.000,00$ & 38,02 & 0,076 \\
\hline$x 6$ & Invalidez Funcional Permanente por Doença (IFPD) & $\mathrm{R} \$ 500.000,00$ & 38,81 & 0,078 \\
\hline$\overline{x 7}$ & Seguro de Assistência Funeral Luxo Individual & $\mathrm{R} \$ \quad 5.500,00$ & 2,37 & 0,431 \\
\hline $\mathbf{x 8}$ & Seguro de Assistência Funeral Luxo Familiar & $\mathrm{R} \$ \quad 5.500,00$ & 6,76 & 1,229 \\
\hline$x 9$ & Seguro de Assistência Funeral Superluxo Individual & $\mathrm{R} \$ \quad 7.000,00$ & 3,02 & 0,431 \\
\hline $\mathbf{x 1 0}$ & Seguro de Assistência Funeral Superluxo Familiar & $\mathrm{R} \$ \quad 7.000,00$ & 8,61 & 1,230 \\
\hline
\end{tabular}

Tabela 2 - Valores por milhar de benefício

Fonte: Autor (2019)

Portanto, a FO ficou:

$$
\begin{gathered}
\operatorname{Min} f(x)=2,626 X_{1}+0,320 X_{2}+0,450 X_{3}+21,804 X_{4}+0,076 X_{5}+0,078 X_{6}+0,431 X_{7}+ \\
1,229 X_{8}+0,431 X_{9}+1,230 X_{10}
\end{gathered}
$$

\subsection{RESTRIÇÕES TÉCNICAS}


Tanto o Whole Life com pagamento até 60 anos quanto o Term Life de 20 anos são produtos com cobertura para morte. Por imposição da Seguradora, ambos possuem um valor mínimo unitário de R\$ 500.000,00. Portanto:

$$
\mathbf{X}_{1}, \mathbf{X}_{2} \geq \mathbf{5 0 0}
$$

Fez uso de uma fórmula básica, prática comum no mercado, para definir o valor mínimo da combinação de ambos. Desta forma:

- Educação do filho:

3000 (valor da mensalidade) X 12 (meses por ano) X 5 (anos previstos para término do ensino médio $)=R \$ 180.000,00$

- Manutenção do padrão de vida:

Como convenção, usa-se 1 (um) ano como período para reestabelecimento, logo:

23000 (renda mensal do cliente) X 12 (meses no ano) $=\mathbf{R} \$ 276.000,00$

$$
500+180+276=R \$ 946.000,00
$$

Desta maneira:

$$
X_{1}+X_{2} \geq 946
$$

Por exigência do cliente, Doenças Graves, Invalidez Permanente Total ou Parcial por Acidente e Invalidez Funcional Permanente por Doença têm valor mínimo de R\$ 200.000,00. Já a Seguradora MA, impõe limite máximo de R $\$ 10.000 .00,00$ ao Invalidez Permanente Total ou Parcial por Acidente, R\$1.000.000,00 ao Invalidez Funcional Permanente por Doença e R \$30.000,00 ao Diária por Incapacidade Temporária com LER e DORT (DIT), sendo assim:

$$
\begin{gathered}
X_{3} \geq \mathbf{2 0 0} \\
X_{4} \leq \mathbf{3 0} \\
X_{5} \geq \mathbf{2 0 0} \\
X_{5} \leq \mathbf{1 0 0 0 0} \\
X_{6} \geq \mathbf{2 0 0} \\
X_{6} \leq \mathbf{1 0 0 0}
\end{gathered}
$$

A família de produtos Seguro de Assistência Funeral possuem um valor único de benefício, R \$ 5.500,00 e R \$ 7.000,00, para Luxo e Superluxo, respectivamente. Só existe a possibilidade da contratação de um da família por apólice, isto posto:

$$
\begin{aligned}
& \mathbf{X}_{7} \leq 1 \\
& \mathbf{X}_{8} \leq 1 \\
& \mathbf{X}_{9} \leq 1 \\
& \mathbf{X}_{10} \leq 1 \\
& \mathbf{X}_{7}+\mathbf{X}_{8}+\mathbf{X}_{9}+\mathbf{X}_{10} \leq 1
\end{aligned}
$$




\section{RESULTADOS OBTIDOS}

Após a modelagem do problema no software LINDO e as iterações necessárias serem feitas, obteve-se o resultado ótimo mostrado na Figura 5.

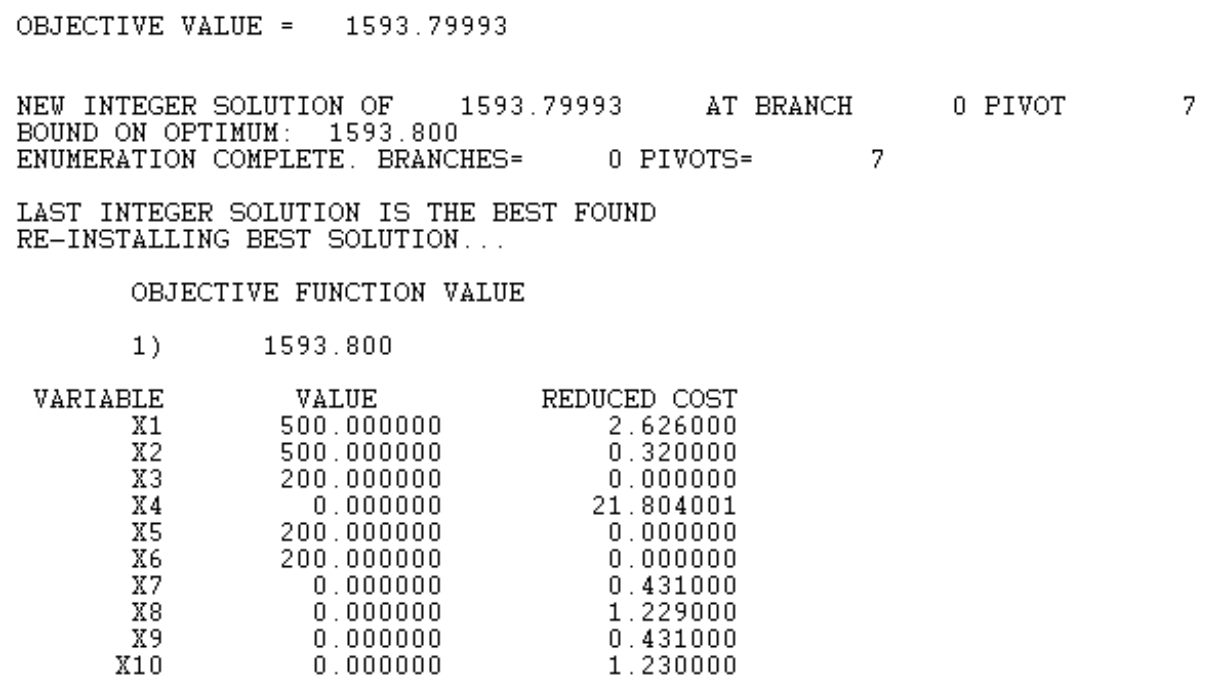

Figura 5 - Resultado ótimo obtido pelo LINDO

Fonte: Autor (2019)

Com isso, a conjuntura da apólice seria:

Whole Life com pagamento até 60 anos - R\$ 500.000,00

Term Life de 20 anos - R\$ 500.000,00

Doenças Graves - R\$ 200.000,00

Invalidez Permanente Total ou Parcial por Acidente - R\$ 200.000,00

Invalidez Funcional Permanente por Doença - R\$ 200.000,00

\section{Custo total: $\mathrm{R} \$ \mathbf{1 . 5 9 3 , 8 0}$}

\section{CONSIDERAÇÕES FINAIS}

A Programação Linear Inteira mostra-se eficiente no que se propôs. Determinou, de forma inequívoca, o menor custo possível dentro das restrições técnicas do modelo. Entretanto, percebe-se que o método foi desnecessário no caso estudado, uma vez que já se conhecia os valores encontrados. Portanto, é possível que não fosse o melhor método para esta situação em particular, porém, é inegável a sua acurácia em eventos propícios.

\section{REFERÊNCIAS BIBLIOGRÁFICAS}

BARBOSA, G.M. Utilização da programação linear na otimização de resultados de produção na empresa. Revista Integração - Universidade São Judas Tadeu. São Paulo. n. 66. p.49-58. 2014.

COLIN, E.C. Pesquisa Operacional: 170 aplicações em Estratégia, Finanças, Logística, Produção, Marketing e Vendas. Rio de Janeiro: LTC, 2007. 
HILliER, F. S.; LIEBERMAN, G. J. Introduçao à Pequisa Operacional. Porto Alegre: Bookmann, 2010.

LOESCH, C.; HEIN, N. Pesquisa Operacional - Fundamentos e Modelos. São Paulo: Saraiva, 2009.

MILHOMEM, D.A. Utilização da Programação Linear e do Método Simplex para a otimização da produção de pães em uma empresa de panificação. Encontro Nacional de Engenharia de Produção. Fortaleza, 2015.

MURTHY. P. Rama. Operations Research (First Edition) - New Age International (P) Limited, Publishers, New Delhi, 2005.

SANTOS, M. Simulação da Operação de um Sistema Integrado de Informações para o atendimento pré-hospitalar de emergência no município do Rio de Janeiro. Dissertação de M.Sc., COPPE/UFRJ, Rio de Janeiro, Brasil, 2013.

SANTOS, M. Proposta de otimização do mix de produção utilizando o Método Simplex: um estudo de caso de uma confecção de moda íntima do município de Cordeiro - RJ. Simpósio de Engenharia de Produção: Lean Cost Management como filosofia global de otimização em organizações. Recife (PE): FBV, 2016.

SANTOS, M. et al. Aplicação da Programação Linear na formulação de uma dieta de custo mínimo: estudo de caso de uma empresa de refeições coletivas no Estado do Rio de Janeiro. Encontro Mineiro de engenharia de Produção. Juiz de Fora, 2017.

SILVA, E.M. et al. Pesquisa Operacional. 3.ed. São Paulo: Editora Atas, 2007.

SUTTO, G. Brasil tem o menor número de pessoas com seguro de vida, mostra estudo. InfoMoney. 02, jan. 2017. Disponível em $<\underline{\text { https://www.infomoney.com.br/minhas-financas/ }}$ noticia/5966445/brasil-tem-menor-numero-pessoas-com-seguro-vida-mostra-estudo $>$. Acesso em: 25, mai. 2019.

TAHA, H.A. Pesquisa Operacional. 8.ed. São Paulo: Pearson Prentice Hall, 2008. 